\title{
ANALYSIS OF QUASISTATIC FRICTIONAL CONTACT PROBLEM WITH SUBDIFFERENTIAL FORM, UNILATERAL CONDITION AND LONG-TERM MEMORY
}

\author{
A. OURAHMOUN ${ }^{1, *}$, B. BOUDERAH ${ }^{1}$, T. SERRAR ${ }^{2}$ \\ ${ }^{1}$ University of M'sila 28000, M'sila LMPA Laboratory, Algeria \\ ${ }^{2}$ University of Setif 1, 19000, Algeria \\ *Corresponding author: ourahmounabbes@yahoo.fr
}

\begin{abstract}
We consider a quasistatic problem which models the contact between a deformable body and an obstacle called foundation. The material is assumed to have a viscoelastic behavior that we model with a constitutive law with long-term memory, thus at each moment of time, the stress tensor depends not only on the present strain tensor, but also on its whole history. In Contact Mechanics, history-dependent operators could arise both in the constitutive law of the material and in the frictional contact conditions. The mathematical analysis of contact models leads to the study of variational and hemivariational inequalities. For this reason a large number of contact problems lead to inequalities which involve history dependent operators, called history dependent inequalities. Such inequalities could be variational or hemivariational and variational hemivariational.

In this paper we derive a weak formulation of the problem and, under appropriate regularity hypotheses, we stablish an existence and uniqueness result. The proof of the result is based on arguments of variational inequalities monotone operators and Banach fixed point theorem.
\end{abstract}

\section{INTRODUCTION}

Contact mechanics still remain a rich domain of research, and the literature devoted to various aspects of the subject is growing. An early attempt at the study of contact problems for elastic viscoelastic materials

Received 2019-12-31; accepted 2020-02-03; published 2020-03-02.

2000 Mathematics Subject Classification. Primary 74M10, 74M15, 49J40.

Key words and phrases. elastic material; frictional contact; unilateral condition; weak solution.

(C)2020 Authors retain the copyrights of their papers, and all open access articles are distributed under the terms of the Creative Commons Attribution License. 
within the mathematical analysis framework was introduced in the pioneering reference works $[6,7,15]$. Further extensions to non convex contact conditions with non-monotone and possible multi-valued constitutive laws led to the active domain of non-smooth mechanic within the framework of the so-called hemivariational inequalities, for a mathematical as well as mechanical treatment we refer to [10]. There is a growing interest in the study of history-dependent inequalities. For instance, a class of variational inequalities with history dependent operators was considered in [15], where abstract existence, uniqueness and regularity results were proved. These results were extended in[18] to a more general class of variational inequalities and were completed in [6] with error estimate and convergence results. Various results on hemivariational and variational-hemivariational inequalities with history dependent operators, formulated in Sobolev-type spaces, could befound in $[7,9]$.

We introduce a new model of frictional contact for viscoelastic materials and to illustrate the use of history dependent variational hemivariational inequality in its variational analysis. Thus, in Section 2 we introduce the contact problem, in which the material's behavior is modeled by a nonlinear viscoelastic constitutive law with long memory, the process is quasistatic, the contact is frictional and the contact conditions are in a subdifferential form with unilateral conditions for the displacement. Then, in Section 3 we list the assumptions on the data and derive the variational formulation of the problem. It is in a form of a historydependent variational-hemivariational inequality in which the unknown is the displacement field.Next in Section 4 we state our main existence and uniqueness result, Theorem (4.2) the proof of the theorem is obtained by using arguments of elliptic variational-hemivariational inequalities and a fixed point result for history dependent operators.

\section{The Contact Model}

The physical setting we consider is the following. A deformable body occupies a domain $\Omega \subset \mathbb{R}^{d}(d=$ 1,2,3 in applications) with outer Lipschitz surface $\Gamma$ that is divided into three disjoint measurable parts $\Gamma_{i}$ $(i=1,2,3)$ such that meas $\left(\Gamma_{1}\right)>0$. Let $[0, T]$ be the time interval of interest, where $T>0$. The body is clamped on $\Gamma_{1} \times(0, T)$ and therefore the displacement field vanishes there. A volume force of density $f_{0}$ acts in $\Omega \times(0, T)$ and surface tractions of density $f_{1}$ act on $\Gamma_{2} \times(0, T)$.

The body is in contact on $\Gamma_{3} \times(0, T)$ with a rigid obstacle, the so-called foundation is in frictional contact. We assume that the process is quasistatic with long term memory and we use (1) as constitutive law. We denote by $\mathbf{u}, \boldsymbol{\sigma}$ and $\varepsilon(u)$ the displacement field, the stress field and the linearized strain tensor, respectively, and let $v$ be the unit outward normal vector to $\Gamma$. Here and below, we sometimes do not indicate explicitly the dependence of various functions on the spatial variable $\mathbf{x} \in \Omega \cup \Gamma$. For a vector field $\mathbf{u}$, we use notation $\mathbf{u}_{v}=\mathbf{u} \cdot v$ and $\mathbf{u}_{\tau}=\mathbf{u}-\mathbf{u}_{v} v$ for the normal and tangential components of $\mathbf{u}$ on $\Gamma$. Similarly, for the stress 
field $\boldsymbol{\sigma}$, its normal and tangential components on the boundary are defined by equalities $\boldsymbol{\sigma}_{v}=(\boldsymbol{\sigma} v) \cdot v$ and $\boldsymbol{\sigma}_{\tau}=\boldsymbol{\sigma} v-\boldsymbol{\sigma}_{v} v$, respectively.

Finally, we use $\mathcal{S}^{d}$ for the space of second order symmetric tensors on $\mathbb{R}^{d}$ and "." will represent the canonical inner product and the Euclidean norm on the spaces $\mathbb{R}^{d}$ and $\mathcal{S}^{d}$, respectively. We also use the following notation:

$$
\begin{gathered}
H=\left(L^{2}(\Omega)\right)^{d}, \mathcal{H}=\left\{\boldsymbol{\sigma}=\left(\sigma_{i j}\right) \mid \sigma_{i j}=\sigma_{j i} \in L^{2}(\Omega), 1 \leq i \leq j \leq d\right\} \\
H_{1}=\{u \in H: \varepsilon(u) \in \mathcal{H}\} \quad ; \mathcal{H}_{1}=\{\sigma \in \mathcal{H} \mid \text { Div } \sigma \in H\}
\end{gathered}
$$

Here $\varepsilon: H_{1} \rightarrow H$ and Div $: H_{1} \rightarrow H$ are the deformation and the divergence operators, respectively, defined by:

$$
\boldsymbol{\varepsilon}(\mathbf{u})=\left(\varepsilon_{i j}(u)\right) \quad ; \quad \varepsilon_{i j}(u)=\frac{1}{2}\left(u_{i, j}+u_{j, i}\right) \quad ; \quad \operatorname{Div} \boldsymbol{\sigma}=\left(\sigma_{i j, j}\right)
$$

The spaces $H, \mathcal{H}, H_{1}$ and $\mathcal{H}_{1}$ are real Hilbert spaces endowed with the canonical inner products given by:

$$
\begin{gathered}
(u, v)_{H}=\int_{\Omega} u_{i} \cdot v_{i} d x \quad,(\sigma, \tau)_{\mathcal{H}}=\int_{\Omega} \sigma_{i j} \cdot \tau_{i j} d x \\
(u, v)_{H_{1}}=(u, v)_{H}+(\varepsilon(u), \varepsilon(v))_{\mathcal{H}} \\
(\sigma, \tau)_{\mathcal{H}_{1}}=(\sigma, \tau)_{\mathcal{H}}+(D i v \sigma, D i v \tau)_{H}
\end{gathered}
$$

We recall that $C$ denotes the class of continuous functions; and $C^{m}, m \in \mathbb{N}^{*}$ the set of m times continuously differentiable functions.

Finally $D(\Omega)$ denotes the set of infinitely differentiable real functions with compact support in $\Omega$; and $W$ ${ }^{m, p}, m \in N, 1 \leq p \leq+\infty$ for the classical Sobolev spaces; and

$$
H_{0}^{m}(\Omega):=\left\{w \in W^{m, 2}(\Omega), w=0 \text { on } \Gamma\right\}, m \geq 1
$$

With these assumptions, the classical formulation or mathematical model which describes the equilibrium of the body in the physical setting above is the following.

Problem P. Find a displacement field $\mathbf{u}: \Omega \times \mathbb{R}_{+} \rightarrow \mathbb{R}^{d}$, a stress field $\boldsymbol{\sigma}: \Omega \times \mathbb{R}_{+} \rightarrow \mathcal{S}^{d}$ and two interface forces $\eta_{v}: \Gamma_{3} \times \mathbb{R}_{+} \rightarrow \mathbb{R}$ and $\xi_{v}: \Gamma_{3} \times \mathbb{R}_{+} \rightarrow \mathbb{R}$ such that

$$
\sigma(t)=\mathcal{A} \varepsilon(u(t))+\int_{0}^{t} \mathcal{B}(t-s) \varepsilon(u(s)) d s \quad \text { in } \Omega \times(0, T)
$$




$$
\begin{aligned}
& \operatorname{Div}(\sigma(t))+f_{0}(t)=0 \quad \text { in } \Omega \times(0, T) \\
& u(t)=0 \quad \text { on } \Gamma_{1} \times(0, T) \\
& \sigma(t) v=f_{2}(t) \quad \text { on } \Gamma_{2} \times(0, T) \\
& u_{v}(t) \leq g \quad, \sigma_{v}(t)+\xi_{v}(t)+\eta_{v}(t) \leq 0 \\
& \left(u_{v}(t)-g\right)\left(\sigma_{v}(t)+\xi_{v}(t)+\eta_{v}(t)\right)=0 \\
& \left|u_{v}(t)\right| \leq F_{m}\left(\int_{0}^{t} u_{v}^{+}(s) d s\right) \\
& \eta_{v}(t)=\left\{\begin{array}{c}
0 \quad \text { if } u_{v}(t) \prec 0 \\
F_{m}\left(\int_{0}^{t} u_{v}^{+}(s) d s\right) \quad \text { if } u_{v}(t) \geq 0
\end{array}\right. \\
& \xi_{v}(t) \in \partial j_{v}\left(u_{v}(t)\right) \quad \text { on } \quad \Gamma_{3} \times(0, T) \\
& \left\{\begin{array}{c}
\left\|\sigma_{\tau}(t)\right\| \leq F_{b}\left(u_{v}(t)\right) \\
-\sigma_{\tau}(t)=F_{b}\left(u_{v}(t)\right) \frac{u_{\tau}(t)}{\left\|u_{\tau}(t)\right\|} \text { if } u_{\tau}(t) \neq 0
\end{array} \text { on } \Gamma_{3} \times(0, T)\right.
\end{aligned}
$$

First, Eq.(2.1) is the constitutive law for viscoelastic materials in which $\mathcal{A}$ represent the elasticity operator and $\mathcal{B}$ represents the relaxation tensor. Various comments and mechanical interpretation related to such kind of equations could be found in $[8,16]$. Equation $(2.2)$ is the equilibrium equation that we use here since we assume that the process is quasistatic. Conditions (2.3) and (2.4) represent the displacement and traction conditions, respectively. Condition (2.5) represents the contact condition in which $g>0, j_{v}$ and $F_{m}$ are given functions and $\partial j_{v}$ represents the Clarke subdifferential of $j_{v}$. Finally, relations $(2.7)$ represent the static version of Coulomb's law of dry friction. Here $F_{b}$ denotes a positive function, the friction bound assumed to depend on the normal displacement $u_{v}$. The contact condition (2.5) represents the trait of novelty of our model. Note that this condition models the contact with a foundation made of a rigid body covered by a layer made of soft material and a thin crust with memory effects.

\section{Variational analysis}

To derive a variational formulation of the problem we use the spaces for the displacement field we use the space

$$
V=\left\{v=(v i) \in H^{1}(\Omega) \mid v=0 \text { on } \Gamma_{1}\right\}
$$


which is a real Hilbert space with inner product $(u, v)_{V}=(\varepsilon(u), \varepsilon(v))_{\mathcal{H}}$ where

$$
(u, v)_{V}=\int_{\Omega} \varepsilon(u) \cdot \varepsilon(v) d x
$$

and associated norm $\|\cdot\|_{V}$.

we consider the space of fourth-order tensor fields

$$
Q_{\infty}=\left\{\mathcal{E}=\left(\mathcal{E}_{i j k l}\right) \mid \mathcal{E}_{i j k l}=\mathcal{E}_{k l i j}=\mathcal{E}_{j i k l} \in L^{\infty}(\Omega)\right\}
$$

which is a real Banach space with norm

$$
\|\mathcal{E}\|_{Q_{\infty}}=\max _{0 \leq i, j, k, l \leq d}\left\|\mathcal{E}_{i j k l}\right\|_{L^{\infty}(\Omega)}
$$

Finally, we use $\mathbb{N}$ for the set of positive integers and $\mathbb{R}_{+}$for the set of nonnegative real numbers. For a normed space $X$, we use the notation $C\left(\mathbb{R}_{+} ; X\right)$ for the space of continuous functions defined on $\mathbb{R}_{+}$with values in $X$.

By the Sobolev trace theorem, we have

$$
\|v\|_{L^{2}\left(\Gamma_{3}, \mathbb{R}^{d}\right)} \leq\|\gamma\|\|v\|_{V}, \forall v \in V
$$

$\|\gamma\|$ being the norm of the trace operator $\gamma: V \rightarrow L^{2}\left(\Gamma_{3}, \mathbb{R}^{d}\right)$

We now list the assumptions on the data and we assume that

1. the elasticity operator $\mathcal{A}: \Omega \times S^{d} \rightarrow \mathcal{S}^{d}$ satisfies the following properties

$$
\left\{\begin{array}{c}
\text { (a) There exists } L_{\mathcal{A}}>0 \text { such that for all } \varepsilon_{1}, \varepsilon_{2} \in \mathcal{S}^{d} \text {,a.e. } x \in \\
\left\|\mathcal{A}\left(x, \varepsilon_{1}\right)-\mathcal{A}\left(x, \varepsilon_{2}\right)\right\| \leq L_{\mathcal{A}}\left|\varepsilon_{1}-\varepsilon_{2}\right| \\
\text { (b) There exists } m>0 \text { such that for all } \varepsilon_{1}, \varepsilon_{2} \in \mathcal{S}^{d} \text {,a.e. } x \in \Omega \\
\left(\mathcal{A}\left(x, \varepsilon_{1}\right)-\mathcal{A}\left(x, \varepsilon_{2}\right)\right) \cdot\left(\varepsilon_{1}-\varepsilon_{2}\right) \geq m\left\|\varepsilon_{1}-\varepsilon_{2}\right\|^{2} \\
\mathcal{A}\left(\cdot, \varepsilon_{1}\right) \text { is measurable on } \Omega \text { for all } \varepsilon \in \mathcal{S}^{d} \\
\mathcal{A}(x, 0)=0 \text { for a.e. } x \in \Omega
\end{array}\right.
$$

2. The relaxation tensor $\mathcal{B}$ is such that

$$
\mathcal{B} \in \mathcal{C}\left(\mathbb{R}_{+}, Q_{\infty}\right)
$$


3. the potential function $j_{v}: \Gamma_{3} \times \mathbb{R} \rightarrow \mathbb{R}$, assumed to satisfy the following conditions

$$
\left\{\begin{array}{c}
\text { (a) } j_{v}(\cdot, r) \text { is measurable on } \Gamma_{3} \text { for all } r \in R \text { and there } \\
\text { exists } \bar{e} \in L^{2}\left(\Gamma_{3}\right) \text { such that } j_{v}(\cdot, \bar{e}) \in L^{1}\left(\Gamma_{3}\right) \\
\text { (b) } j_{v}(x, \cdot) \text { is locally Lipschitz on } \mathbb{R} \text { for a.e. } x \in \Gamma_{3} \\
\text { (c) }\left|j_{v}(x, r)\right| \leq \bar{c}_{0}+\bar{c}_{1}|r| \text { for a.e. } x \in \Gamma_{3} \text { and } \\
\text { for all } r \in \mathbb{R} \text { with } \bar{c}_{0}, \bar{c}_{1} \geq 0
\end{array}\right.
$$

Next, we assume that the penetration bound $g: \Gamma_{3} \rightarrow \mathbb{R}$, the memory function $F_{m}: \Gamma_{3} \times \mathbb{R} \rightarrow \mathbb{R}+$ and the friction bound $F_{b}: \Gamma_{3} \times \mathbb{R} \rightarrow \mathbb{R}$ are assumed to satisfy the following conditions.

$$
g \in L^{2}\left(\Gamma_{3}\right), g(x) \geq 0 \text { a.e.on } \Gamma_{3} .
$$

And

(a)There exists $L_{F_{m}}>0$ such that $\left|F_{m}\left(x, r_{1}\right)-F_{m}\left(x, r_{1}\right)\right| \leq L_{F_{m}}\left|r_{1}-r_{2}\right|$

for all $r_{1}, r_{2} \in \mathbb{R}$, a.e. $x \in \Gamma_{3}$

(b) $F_{m}(\cdot, r)$ is measurable on $\Gamma_{3}$ for all $r \in \mathbb{R}$

(c) $x \rightarrow F_{m}(x, 0) \in L^{2}\left(\Gamma_{3}\right)$

(a)There exists $L_{F_{b}}>0$ such that $\left|F_{b}\left(x, r_{1}\right)-F_{b}\left(x, r_{1}\right)\right| \leq L_{F_{b}}\left|r_{1}-r_{2}\right|$

for all $r_{1}, r_{2} \in \mathbb{R}$, a.e. $x \in \Gamma_{3}$

(b) $F_{b}(\cdot, r)$ is measurable on $\Gamma_{3}$, for all $r_{1}, r_{2} \in \mathbb{R}$, a.e. $x \in \Gamma_{3}$

(c) $F_{b}(x, r)=0$ for $r \leq 0, F_{b}(x, r)>0$ for all $r>0$ a.e. $x \in \Gamma_{3}$

We also assume that the densities of body forces and surface tractions have the regularity

$$
f_{0} \in C\left(\mathbb{R}_{+} ; L^{2}\left(\Omega ; \mathbb{R}^{d}\right)\right), \quad f_{2} \in C\left(\mathbb{R}_{+} ; L^{2}\left(\Gamma_{2} ; \mathbb{R}^{d}\right)\right)
$$

and, finally, we assume the smallness condition

$$
L_{F_{b}}\|\gamma\|+\alpha j_{v}<m_{F}
$$

We now introduce the set of the admissible displacement fields $U \subset V$ and the function $f: \mathbb{R}_{+} \rightarrow V^{\prime}$ defined by

$$
\left\{\begin{array}{c}
U=\left\{\mathbf{v} \in V \mid \mathbf{v}_{v} \leq g \text { on } \Gamma_{3}\right\} \\
\langle f(t), \mathbf{v}\rangle_{V^{\prime} \times V}=\left(f_{0}(t), \mathbf{v}\right)_{\left(L^{2}(\Omega), \mathbb{R}^{d}\right)}+\left(f_{2}(t), \mathbf{v}\right)_{\left(L^{2}\left(\Gamma_{3}\right), \mathbb{R}^{d}\right)}, \text { for all } \mathbf{v} \in \mathbf{V}, \mathbf{t} \in \mathbb{R}_{+}
\end{array}\right.
$$


Assume now that $(\mathbf{u}, \boldsymbol{\sigma})$ represents a couple of regular functions which satisfy $(2.1)-(2.6)$ and let $t \in \mathbb{R}_{+}$, $\mathbf{v} \in U$. We perform an integration by parts, split the surface integral on three integrals on $\Gamma_{1}, \Gamma_{2}$ and $\Gamma_{3}$, and use the equalities $(2.2)-(2.4)$ to deduce that

$$
\begin{gathered}
\int_{\Omega} \sigma(t) \cdot(\varepsilon(\mathbf{v})-\varepsilon(u(t))) d x=\int_{\Omega} f_{0}(t) \cdot(\mathbf{v}-u(t)) d x \\
+\int_{\Gamma_{2}} f_{2}(t) \cdot(\mathbf{v}-u(t)) d \Gamma+\int_{\Gamma_{3}} \sigma_{v}(t)\left(\mathbf{v}_{v}-u_{v}(t)\right) d \Gamma+\int_{\Gamma_{3}} \sigma_{\tau}(t)\left(\mathbf{v}_{\tau}-u_{\tau}(t)\right) d \Gamma
\end{gathered}
$$

Next, we use the contact boundary condition (2.5), the definition (3.12) and the definition of the Clarke subdifferential to obtain that

$$
\begin{gathered}
\int_{\Gamma_{3}} \sigma_{v}(t)\left(\mathbf{v}_{v}-u_{v}(t)\right) d \Gamma+\int_{\Gamma_{3}} F_{m}\left(\int_{0}^{t} u_{v}^{+}(s) d s\right)\left(\mathbf{v}_{v}^{+}-u_{v}^{+}(t)\right) d \Gamma \\
+\int_{\Gamma_{3}} j_{v}^{0}\left(u_{v}(t), \mathbf{v}_{v}-u_{v}(t)\right) d \Gamma \geq 0
\end{gathered}
$$

Note that here and below we use notation $j_{v}^{0}\left(r_{1} ; r_{2}\right)$ for the generalized directional derivative of $j_{v}$ at $r_{1}$ in the direction $r_{2}$, see $[1,2]$ for details.

On the other hand, the friction law (2.6) yields

$$
\int_{\Gamma_{3}} \sigma_{\tau}(t)\left(\mathbf{v}_{\tau}-u_{\tau}(t)\right) d \Gamma+\int_{\Gamma_{3}} F_{b}\left(u_{v}(t)\left(\left\|v_{\tau}\right\|-\left\|u_{\tau}(t)\right\|\right) d \Gamma \geq 0\right.
$$

We now combine equality (3.13) with inequalities (3.14), (3.15) to deduce that

$$
\begin{gathered}
\int_{\Omega} \sigma(t) \cdot(\varepsilon(\mathbf{v})-\varepsilon(u(t))) d x+\int_{\Gamma_{3}} F_{b}\left(u_{v}(t)\left(\left\|v_{\tau}\right\|-\left\|u_{\tau}(t)\right\|\right) d \Gamma+\right. \\
\int_{\Gamma_{3}} F_{m}\left(\int_{0}^{t} u_{v}^{+}(s) d s\right)\left(\mathbf{v}_{v}^{+}-u_{v}^{+}(t)\right) d \Gamma+\int_{\Gamma_{3}} j_{v}^{0}\left(u_{v}(t), \mathbf{v}_{v}-u_{v}(t)\right) d \Gamma \\
\geq \int_{\Omega} f_{0}(t) \cdot(\mathbf{v}-u(t)) d x+\int_{\Gamma_{2}} f_{2}(t) \cdot(\mathbf{v}-u(t)) d \Gamma
\end{gathered}
$$

Finally, we substitute the consitutive law (2.1) in (3.15) and use notation (3.12) to obtain the following variational formulation of Problem $\mathcal{P}$, in terms of displacement.

Problem $\mathcal{P V}$ Find a displacement field $u: \mathbb{R}_{+} \rightarrow U$ such that

The unique solvability of Problem $\mathcal{P} \mathcal{V}$ is given by the following existence and uniqueness result, that we state here and prove in the next section.

Theorem 3.1 Assume that (3.7)-(3.11) hold. Then, Problem $\mathcal{P V}$ has a unique solution $u \in C\left(\mathbb{R}_{+} ; U\right)$. 
We end this section with some remarks on the weak solvability of the contact problem $\mathbf{P}$.

First, a couple of functions $(u, \sigma)$ defined on the positive real line $\mathbb{R}_{+}$with values on the product space $V \times Q$ is called a weak solution to Problem $\mathbf{P}$ if $u$ is a solution of the variational problem $\mathcal{P} \mathcal{V}$ and $\sigma$ satisfies the constitutive law (2.1).

We conclude that, under the assumption of Theorem 8.1, Problem $\mathbf{P}$ has a unique weak solution. Moreover, the solution has the regularity $u \in C\left(\mathbb{R}_{+} ; U\right)$ and $\sigma \in C\left(\mathbb{R}_{+} ; Q\right)$.

Next, recall that Theorem 8.1 provides the weak solvability of the contact problem $\mathbf{P}$ under the smallness assumption (24) involving the friction bound $F_{b}$, and the normal compliance potential $j_{v}$. Finally, note that the unknowns $\eta_{v}$ and $\xi_{v}$ of Problem $\mathbf{P}$ cannot be recovered since they cannot be computed when the solution $u$ of Problem $\mathbf{P}$ is known.Actually, these unknowns represent interface forces and, as usual in solving contact problems with unilateral constraints, we do not have information neither on the uniqueness of these functions and on their regularity.

\section{An Existence and Uniqueness Result}

We present in this section an abstract result on history-dependent variational-hemivariational inequalities that we shall use to prove the unique solvability of Problem $\mathcal{P} \mathcal{V}$. For more details on the material presented in this section, we send the reader to $[1,2]$.

Theorem 4.1.Let $X$ be a reflexive Banach space and $Y$ be a normed space. We denote by $X^{\prime}$ the dual of $X$ and by $\langle\cdot, \cdot\rangle_{X^{\prime} \times X}$ the duality pairing of $X$ and $X^{\prime}$. Let $K$ be a subset of $X$ and $A: X \rightarrow X{ }^{\prime}, \Psi:$ $C\left(\mathbb{R}_{+} ; X\right) \rightarrow C\left(\mathbb{R}_{+} ; Y\right)$ be given operators, consider also a function $\phi: Y \times K \times K \rightarrow \mathbb{R}$, a locally Lipschitz function $j: X \rightarrow \mathbb{R}$ and a function $f: \mathbb{R}_{+} \rightarrow X^{\prime}$. With these data we consider the problem of finding a function $u: \mathbb{R}_{+} \rightarrow U$ such that, for each $t \in \mathbb{R}_{+}$, the following inequality holds:

$$
\begin{gathered}
\langle A u(t), v-u(t))\rangle+\phi((\Psi u)(t), u(t), v)-\phi((\Psi u)(t), u(t), u(t)) \\
\quad+j^{0}(u(t), v-u(t)) \geq\langle f(t), v-u(t)\rangle, \quad \text { for all } v \in K
\end{gathered}
$$

In the study of (4.1), we assume the following hypotheses.

$K$ is a nonempty, closed and convex subset of $X$.

$A: X \rightarrow X^{\prime}$ is an operator such that

$$
\left\{\begin{array}{c}
(a) A \text { is pseudomonotone and there exist } \\
\alpha_{A}>0, \beta_{A}, \gamma_{A} \in \mathbb{R} \text { and } u_{0} \in K \text { such that: } \\
\left\langle A v, v-u_{0}\right\rangle \geq \alpha_{A}\|v\|_{X}^{2}-\beta_{A}\|v\|_{X}^{2}-\gamma_{A} \text { for all } v \in X . \\
(b) A \text { is strongly monotone,i.e., there exists } m_{A}>0 \text { such that } \\
\left\langle A v_{1}-A v_{2}, v_{1}-v_{2}\right\rangle \geq m_{A}\left\|v_{1}-v_{2}\right\|_{X}^{2} \quad, \forall v_{1}, v_{2} \in X
\end{array}\right.
$$


$\phi: Y \times K \times K \rightarrow \mathbb{R}$ is a function such that

$$
\left\{\begin{array}{c}
(a) \phi(y, u, \cdot) \text { is convex and l.s.c. on } \mathrm{K}, \text { for all } y \in Y, u \in K \\
(b) \text { there exist } \alpha_{\phi}, \beta_{\phi}>0 \text { such that } \\
\phi\left(y_{1}, u_{1}, v_{2}\right)-\phi\left(y_{1}, u_{1}, v_{1}\right)+\phi\left(y_{2}, u_{2}, v_{1}\right)-\phi\left(y_{2}, u_{2}, v_{2}\right) \leq \\
\alpha_{\phi}\left\|u_{1}-u_{2}\right\|_{X}\left\|v_{1}-v_{2}\right\|_{X}+\beta_{\phi}\left\|y_{1}-y_{2}\right\|_{Y}\left\|v_{1}-v_{2}\right\|_{X} \\
\text { for all } y_{1}, y_{2} \in Y, u_{1}, u_{2}, v_{1}, v_{2} \in K
\end{array}\right.
$$

$j: X \rightarrow \mathbb{R}$ is a function such that

$$
\begin{gathered}
(a) j \text { is locally Lipschitz } \\
(b)\|\partial j(v)\|_{X^{\prime}} \leq c_{0}+c_{1}\|v\|_{X}, \text { for all } v \in V, c_{0}, c_{1} \geq 0 \\
(c) \text { there exists } \alpha_{j}>0 \text { such that } \\
j^{0}\left(v_{1}, v_{2}-v_{1}\right)-j^{0}\left(v_{2}, v_{1}-v_{2}\right) \leq \alpha_{j}\left\|v_{1}-v_{2}\right\|_{X}^{2}, \text { for all } v_{1}, v_{2} \in X \\
\left\{\begin{array}{c}
\text { For any } n \in \mathbb{N}, \text { there exists } s_{n}>0 \text { such that } \\
\left\|\left(\Psi u_{1}\right)(t)-\left(\Psi u_{2}\right)(t)\right\|_{Y} \leq s_{n} \int_{0}^{t}\left\|u_{1}(s)-u_{2}(s)\right\| d s \\
\text { for all } u_{1}, u_{2} \in C\left(\mathbb{R}_{+} ; X\right), \text { for all } t \in[0, n] . \\
\alpha_{\varphi}+\alpha_{j}<m_{A} \quad ; \alpha_{\varphi}<\alpha_{j} \\
f \in C\left(\mathbb{R}_{+} ; X^{*}\right)
\end{array}\right.
\end{gathered}
$$

Note that an operator $\Psi$ which satisfies condition (4.5) is called a history dependent operator. Inequality (4.1) is governed both by the function $\phi$ which is assumed to be convex with respect its second argument and by the function $j$ which is locally Lipschitz and could be nonconvex. Therefore, this inequality is a variational-hemivariational inequality. In addition, the function $\phi$ in (4.1) depends on the operator $\Psi$, assumed to be history-dependent.For this problem we have the following existence and uniqueness result.

Theorem 4.2. Let $X$ be a reflexive Banach space, $Y$ a normed space, and assume that (4.2)-(4.7) hold. Then, inequality (4.1) has a unique solution $u \in C\left(\mathbb{R}_{+} ; K\right)$.

The proof of is obtained by using arguments of elliptic variational-hemivariational inequalities and a fixed point result for history dependent operators.

Proof (Theorem 4.1) We start by defining the operators $A: V \rightarrow V^{\prime}, \mathcal{F}: C\left(\mathbb{R}_{+} ; V\right) \rightarrow C\left(\mathbb{R}_{+} ; Q \times L^{2}\left(\Gamma_{3}\right)\right)$ and the functions $\phi: L^{2}\left(\Gamma_{3}\right) \times V \times V \rightarrow \mathbb{R}$ and $j: V \rightarrow \mathbb{R}$ by

$$
(A \mathbf{u}, \mathbf{v})=\int_{\Omega} \mathcal{F} \varepsilon(u) \cdot \varepsilon(v) d x \text { for all } \mathbf{u}, \mathbf{v} \in V
$$




$$
\begin{aligned}
& (\mathcal{F} \mathbf{u})(t)=\left(\int_{\Omega} \mathcal{B}(t-s) \varepsilon(\mathbf{u}(s)) d s, F_{m}\left(\int_{0}^{t} u_{v}^{+}(s) d s\right)\right) \\
& \quad \text { for all } \mathbf{u} \in C\left(\mathbb{R}_{+} ; V\right), t \in \mathbb{R}_{+} \\
& \varphi(\xi, \mathbf{u}, \mathbf{v})=\left(\xi_{1}, \varepsilon(v)\right)_{Q}+\left(\xi_{2}, v_{v}^{+}\right)_{L^{2}\left(\Gamma_{3}\right)}+\left(F_{b}\left(u_{v}\right),\left\|v_{\tau}\right\|\right)_{L^{2}\left(\Gamma_{3}\right)} \\
& \text { for all } \xi=\left(\xi_{1}, \xi_{2}\right) \in Q \times L^{2}\left(\Gamma_{3}\right), \mathbf{u}, \mathbf{v} \in V \\
& j(v)=\int_{\Gamma_{3}} j_{v}\left(\mathbf{v}_{v}\right) d \Gamma ; \text { for all } \mathbf{v} \in \mathbf{V} .
\end{aligned}
$$

Then, it is easy to see that Problem $\mathcal{P} \mathcal{V}$ is equivalent to the problem of finding a function $u: \mathbb{R}_{+} \rightarrow U$ such that for each $t \in \mathbb{R}_{+}$, the following inequality holds:

$$
\begin{gathered}
\langle A \mathbf{u}(t), \mathbf{v}-\mathbf{u}(t)\rangle+\varphi((\mathcal{F} \mathbf{u})(t), \mathbf{u}(t), \mathbf{v})-\varphi((\mathcal{F} \mathbf{u})(t), \mathbf{u}(t), \mathbf{u})+ \\
\left.j^{0}(u(t), \mathbf{v}-\mathbf{u}(t)) \geq\langle f(t), \mathbf{v}-\mathbf{u}(t))\right\rangle \quad, \text { for all } \mathbf{v} \in V
\end{gathered}
$$

To solve this problem, we use Theorem 4.1 with $X=V, Y=L^{2}\left(\Gamma_{3}\right)$ and $K=U$ and, to this end, we check in what follows that assumptions (4.2)-(4.7) hold. We use arguments similar to those used in our previous works [8, 9] and, for this reason, we skip the details and we resume the proof as follows. First, we note that assumption (3.7) and definition (3.12) imply (4.2). Next, a simple calculation based on the definition (4.5) of the operator $A$ and the properties (3.4) of the elasticity operator show that (4.2) holds with $m A$ $=\alpha_{A}=m_{\mathcal{F}}$. Moreover, using assumption (3.9) and the trace inequality (3.2), it is easy to see that the function $\phi$ defined by (4.5) satisfies condition (4.3) with $\alpha_{\phi}=L_{F_{b}}\|\gamma\|$. On the other hand, assumption (3.6) on the function $j_{v}$ and definition (4.8) show that condition (4.4) holds with $\alpha_{j}=\alpha_{j_{v}}$. And, a simple calculation based on assumptions (3.5), (3.9) imply that the operator (4.9) is a history-dependent operator, i.e., it satisfies condition (4.5). Now, keeping in mind that $m_{A}=\alpha_{A}=m_{\mathcal{F}, \alpha_{\phi}}=L_{F_{b}}\|\gamma\|$ and $\alpha_{j}=\alpha$ $j_{v}$, we easily deduce that the smallness assumption (3.11) shows that conditions (4.6) hold, too. Finally, we note that regularity (3.9) on the densites of the body forces and tractions combined with definition (3.12) show that condition (4.7) is satisfied. We are now in a position to use Theorem 4.2 to deduce the existence of a unique function $u \in C\left(\mathbb{R}_{+} ; U\right)$ such that (4.12) holds, for each $t \in \mathbb{R}_{+}$. And, using notation (4.8)-(4.12), we deduce that $u$ is the unique solution to Problem $\mathcal{P} \mathcal{V}$ which concludes the proof.

Conflicts of Interest: The author(s) declare that there are no conflicts of interest regarding the publication of this paper. 


\section{REFERENCES}

[1] Adly, S. Ernst, E., Thera, M.: Stability of the solution set of non-coercive variational inequalities. Commun. Contemp. Math. 4 (2002), 145-160.

[2] Barbu, V. Nonlinear Semigroups and Differential Equations in Banach Spaces; Springer: Heidelberg, Germany, 1976.

[3] Brézis, H. Problèmes unilatéraux. J. Math. Pures Appl. 51 (1972), 1-168.

[4] Carstensen, C., Gwinner, J.: A theory of discretization for nonlinear evolution inequalities applied to parabolic Signorini problems. Ann. Mat. Pura Appl. 177 (1999), 363-394.

[5] Chau, O. Analyse variationnelle et numérique en mécanique du contact. Thesis, Perpignan, June 2000.

[6] Ciarlet, P.G.: Mathematical Elasticity, vol. I: Three-Dimensional Elasticity. North-Holland, Amsterdam, 1988.

[7] Duvaut, G., Lions, J.L.: Les Inéquations en Mécanique et en Physique. Dunod, Paris, 1972.

[8] Eck, Ch., Jarusek, J., Krbec, M.: Unilateral Contact Problems, Variational Methods and ExistenceTheorems. Monographs \& Texbooks in Pure \& Applied Mathematics, vol. 270, Chapman and Hall, London, 2005.

[9] Glowinski, R Numerical Methods for Nonlinear Variational Problems. Springer, Berlin, 1984.

[10] Goeleven, D, Motreanu, D., Dumont, Y., Rochdi, M.: Variational and Hemivariational Inequalities,Theory, Methods and Applications, Volume I: Unilateral Analysis and Unilateral Mechanics. Kluwer, Dordrecht, 2003.

[11] Han, W, Sofonea, M.: Evolutionary variational inequalities arising in viscoelastic contact problems. SIAM J. Numer. Anal. 38 (2000), 556-579. .

[12] Lions, J.L. Quelques méthodes de résolution des problèmes aux limites non linéaires. Dunod et Gauthier-Villars, Paris, 1969.

[13] Lions, J., Magenes, E.: Problèmes aux limites non homogènes et applications, vol. 1. Dunod, Paris, 1968.

[14] Matei, A, Sofonea, M.: Variational Inequalities with Applications: A Study of Antiplane Frictional Contact Problems, Advances in Mechanics and Mathematics, vol. 18. Springer, Berlin, 2009.

[15] Kikuchi, N., Oden, J.T. Contact Problems in Elasticity. SIAM, Philadelphia, 1988.

[16] Necas, J., Hlavácek, I. Mathematical Theory of Elastic and Elastoplastic Bodies: An Introduction.Elsevier, Amsterdam, 1981.

[17] Panagiotopoulos, P.D. Inequality Problems in Meechanics and Applications. Birkhäuser, Basel, 1985.

[18] Panagiotopoulos, P.D. Inequality Problems in Meechanics and Applications. Birkhäuser, Basel, 1985.

[19] Sofonea, M., Han, W., Migórski, S. Numerical analysis of history-dependent variational inequalities with applications to contact problems. Eur. J. Appl. Math. 26 (2015), 427-452.

[20] Sofonea, M., Matei, A. History-dependent quasivariational inequalities arising in contact mechanics. Eur. J. Appl. Math. 22 (2011), 471-491.

[21] Sofonea, M., Matei, A.Mathematical Models in Contact Mechanics, London Mathematical Society Lecture Note Series, vol. 398. Cambridge University Press, Cambridge, 2012.

[22] Sofonea, M., Migórski, S. A class of history-dependent variational-hemivariational inequalities. Nonlinear Differ. Equ. Appl. $23(2016), 38$.

[23] Sofonea, M., Xiao, Y. Fully history-dependent quasivariational inequalities in contact mechanics. Appl. Anal. 95 (2016), $2464-2484$.

[24] Zeidler, E.Nonlinear Functional Analysis and Its Applications, II/A, Linear Monotone Operators. Springer, Berlin, 1997. 